\title{
Light Scattering Study of the Diffusion of Interacting Particles
}

\author{
By Robert Finsy, ${ }^{*}$ Annie Devriese and Hendrik LeKkerkerker \\ Fakulteit van de Wetenschappen, Vrije Universiteit Brussel, \\ Pleinlaan 2, B-1050 Brussels, Belgium
}

Received 19th July, 1979

\begin{abstract}
The diffusion coefficient of a water-in-oil microemulsion has been measured as a function of the concentration of the suspended particles using photon correlation techniques. The data have been analysed using an extension of the thermo-hydrodynamic theory of G. K. Batchelor to the case of a potential of mean force between particles which consists of a hard-core repulsion and an attractive part. The parameters for this potential are consistent with those obtained from the osmotic compressibility as determined by static light scattering.
\end{abstract}

\section{INTRODUCTION}

The understanding of suspensions of interacting particles has recently been substantially advanced by the application of modern liquid state theories. In particular, static properties of these systems have been successfully interpreted in terms of potentials of mean force between the suspended particles. ${ }^{1-3}$

Interesting results for the dynamics of these systems have been obtained using quasi-elastic light scattering. ${ }^{4-11}$ This technique provides information about the diffusion coefficient $D$ of the suspended particles, which depends markedly on the concentration. The quantitative interpretation of these data has been confused by the fact that even for hard spheres the recent literature contains a number of widely different theoretical results. ${ }^{12-25}$ Basically two different approaches to the problem can be distinguished. The first starts from a Smoluchowsky equation for the $N$ particle probability distribution in configuration space. ${ }^{26-28}$ This description must then be reduced to a closed equation for the one-body density which contains the diffusion coefficient. The second, much simpler, approach is based on an extension of the Einstein argument ${ }^{29,30}$ relating mobility and diffusion to the case of interacting particles. The problem that then remains is the calculation of the density dependence of the mobility and the osmotic compressibility. Recently, Felderhof ${ }^{24}$ has shown by explicit calculation that the results obtained from the $N$-particle Smoluchowsky equation are in complete agreement with those Batchelor obtained from the generalized Einstein argument. He also pointed out the omissions of previous authors which led to the various different results.

In this paper, measurements of the diffusion coefficient of a model system of interacting Brownian particles as a function of their concentration are reported. The observed decrease in $D$ with concentration is analysed in terms of an extension of the Batchelor approach to the case of an interparticle potential which contains, in addition to a hard-core repulsion, an attractive part. The parameters for this potential are consistent with the results obtained from the osmotic compressibility as determined by static light scattering. ${ }^{1}$ 


\section{THEORETICAL}

Consider a system of suspended particles with a number density $n$. Local differences in this density will be smoothed out by the process of diffusion. This tendency can be described by the diffusion equation ${ }^{29}$

$$
\frac{\partial n(\boldsymbol{r}, t)}{\partial t}=D \nabla^{2} n(\boldsymbol{r}, t)
$$

Einstein ${ }^{30}$ has shown that the diffusion coefficient $D$ in eqn (1) can be written as

$$
D=b\left(\frac{\partial \Pi}{\partial n}\right)_{T, P}
$$

where $b$ is the mobility of the suspended particles and $\Pi$ is their osmotic pressure.

At infinite dilution, where all interactions between the particles can be neglected, the osmotic pressure is given by the Van't Hoff equation ${ }^{31}$

$$
\Pi_{0}=n k_{\mathrm{B}} T,
$$

where $k_{\mathrm{B}}$ is the Boltzmam constant, and for spherical particles the mobility reduces to the Stokes ${ }^{32}$ expression

$$
b_{0}=\frac{1}{6 \pi \eta a_{\mathrm{H}}} .
$$

Here $a_{\mathrm{H}}$ is the hydrodynamic radius of the suspended particles and $\eta$ is the shear viscosity of the suspending medium. Substituting eqn (3) and (4) in eqn (2) one obtains the Stokes-Einstein ${ }^{33}$ expression for the diffusion coefficient

$$
D_{0}=\frac{k_{\mathrm{B}} T}{6 \pi \eta a_{\mathrm{H}}} .
$$

In general, however, there will be both static and hydrodynamic interactions between the particles affecting the osmotic pressure ${ }^{34}$ and the mobility, ${ }^{35}$ respectively. At low concentrations these effects can be taken into account by expanding $\Pi$ and $b$ in a power series of the concentration

$$
\begin{array}{r}
\Pi=k_{\mathrm{B}} T\left[n+B_{2} n^{2}+\ldots\right] \\
b=\frac{1}{6 \pi \eta a_{\mathrm{H}}}\left[1+\lambda \phi_{\mathrm{H}}+\ldots\right] .
\end{array}
$$

Here $B_{2}$ is the second osmotic virial coefficient and $\phi_{\mathrm{H}}$ is the hydrodynamic volume fraction

$$
\phi_{\mathrm{H}}=\frac{4 \pi a_{\mathrm{H}}^{3}}{3} n .
$$

The coefficient $B_{2}$ can be related to the potential $W_{12}(r)$ of mean force between two particles in the medium ${ }^{34}$

$$
B_{2}=-2 \pi \int_{0}^{\infty}\left[\exp \left(-W_{12} / k_{\mathrm{B}} T\right)-1\right] r^{2} \mathrm{~d} r .
$$


The correct form of the coefficient $\lambda$ in eqn (7) can be obtained from the work of Batchelor ${ }^{35}$

$$
\begin{gathered}
\lambda=\frac{1}{2}+\frac{3}{a_{\mathrm{H}}^{3}} \int_{0}^{a_{\mathrm{H}}}\left[\exp \left(-W_{12} / k_{\mathrm{B}} T\right)-1\right] r^{2} \mathrm{~d} r+\frac{3}{a_{\mathrm{H}}^{2}} \int_{a_{\mathrm{H}}}^{\infty}\left[\exp \left(-W_{12} / k_{\mathrm{B}} T\right)-1\right] r \mathrm{~d} r+ \\
\frac{1}{a_{\mathrm{H}}^{3}} \int_{2 a_{\mathrm{H}}}^{\infty} \exp \left(-W_{12} / k_{\mathrm{B}} T\right)\left[A(r)+2 B(r)-3\left(1+\frac{a_{\mathrm{H}}}{r}\right)\right] r^{2} \mathrm{~d} r .
\end{gathered}
$$

In eqn (9) $b_{0} A(r)$ is the mobility of a pair of suspended particles separated by a distance $r$ along the line connecting their centres and $b_{0} B(r)$ is the mobility perpendicular to this line. Exact expressions for $A(r)$ and $B(r)$ have been obtained by Stimson and Jeffery ${ }^{36}$ and Goldman et al., ${ }^{37}$ respectively. In the derivation of eqn (9) it has been assumed that the suspended particles cannot penetrate into one another beyond their hydrodynamic radius $a_{\mathrm{H}}$, i.e., the minimum distance of approach, which could be called the hard-sphere diameter $2 a_{\mathrm{HS}}$, is assumed $\geqslant 2 a_{\mathrm{H}}$.

The results given in eqn (6)-(9) together with eqn (2) allow one to calculate the concentration dependence of the diffusion coefficient for a given potential subject to the above limitation.

We now briefly outline how the diffusion coefficient can be determined by quasielastic light scattering. ${ }^{38,} 39$ Photon correlation techniques allow one to measure the autocorrelation function of the intensity of the scattered light given by

$$
G_{2}(\tau)=\left\langle I_{s}(0) I_{s}(\tau)\right\rangle .
$$

Since the number of scattering particles is large the scattered light will show Gaussian statistics so that the normalized intensity autocorrelation function satisfies the following relation ${ }^{40}$

$$
g_{2}(\tau)=\frac{G_{2}(\tau)}{\left\langle I_{\mathrm{s}}\right\rangle^{2}}=1+C\left|g_{1}(\tau)\right|^{2} .
$$

Here $\left|g_{1}(\tau)\right|$ is the modulus of the normalized electrical field autocorrelation function

$$
\left|g_{1}(\tau)\right|=\frac{\left|\left\langle E_{\mathrm{s}}(t) E_{\mathrm{s}}^{*}(t+\tau)\right\rangle\right|}{\left\langle I_{\mathrm{s}}\right\rangle}
$$

and $C$ is a constant for a given experiment.

Assuming that the scattering is due to $N$ identical spherical particles which are small compared with the wavelength $\lambda$ of the light in the medium, the electric field of singlescattered light can be written as

$$
E_{\mathrm{s}}(t)=A \exp \left(\mathrm{i} \omega_{0} t\right) \sum_{j=1}^{N} \exp \left[\mathrm{i} \boldsymbol{q} \cdot \boldsymbol{r}_{j}(t)\right] .
$$

Here $q$ is the scattering vector which is related to the scattering angle $\theta$ by

$$
q=|q|=\frac{4 \pi}{\lambda} \sin \frac{1}{2} \theta,
$$

$A$ is the amplitude of the wave scattered by one particle, $\operatorname{et}(t)$ denotes the position of the $j$ th particle and $\omega_{0}$ is the circular frequency of the incident light.

Substituting the above result for the electric field of the scattered light in eqn (11) one obtains

$$
\left|g_{1}(\tau)\right|=\frac{F(q, \tau)}{S(q)}
$$


where

$$
\begin{aligned}
F(\boldsymbol{q}, \tau) & =\frac{1}{N}\left\langle\sum_{i=1}^{N} \sum_{j=1}^{N} \exp \left[\mathrm{iq} \cdot\left(\boldsymbol{r}_{i}(0)-\boldsymbol{r}_{j}(\tau)\right)\right]\right\rangle \\
& =\frac{1}{N}\left\langle n(\boldsymbol{q}, 0) n^{*}(\boldsymbol{q}, \tau)\right\rangle
\end{aligned}
$$

and

$$
S(q)=F(q, 0) .
$$

In eqn (13) $n(\boldsymbol{q}, \tau)$ is the $\boldsymbol{q}$ th spatial Fourier component of the number density $n(\boldsymbol{r}, \tau)$. According to the Onsager regression hypothesis ${ }^{41}$ the time dependence of $n(q, \tau)$ can be obtained from the diffusion equation [eqn (1)] yielding

$$
\langle n(\boldsymbol{q}, \tau)\rangle_{0}=n(\boldsymbol{q}, 0) \exp \left(-D q^{2} \tau\right) \quad(\tau>0)
$$

where $\langle\ldots\rangle_{0}$ signifies a conditional average with $n(q, 0)$ as initial value. Substituting this time dependence in eqn (13) one obtains

$$
\begin{aligned}
\boldsymbol{F}(\boldsymbol{q}, \tau) & =\frac{1}{N}\left\langle|n(\boldsymbol{q}, 0)|^{2}\right\rangle \exp \left(-D q^{2} \tau\right) \\
& =S(\boldsymbol{q}) \exp \left(-D q^{2} \tau\right) .
\end{aligned}
$$

Using the results expressed by eqn (14) and (12) in eqn (10) one finally finds that

$$
g_{2}(\tau)=1+C \exp \left(-2 D q^{2} \tau\right) \text {. }
$$

This expression establishes the link between diffusion coefficients and quasi-elastic light scattering.

\section{EXPERIMENTAL}

The investigated system is a water-in-oil type microemulsion ${ }^{42}$ composed of water (twice distilled), potassium oleate [prepared from oleic acid (Merck) and potassium hydroxide (Merck)], benzene and butanol (Merck, pro analysis). The suspended droplet sconsist of a core (water+butanol) covered with a mixed surface layer of potassium oleate (soap) + butanol. The continuous phase is a mixture of benzene+butanol. The particle size is largely determined by the ratio of the number of water molecules $N_{\mathrm{W}}$ to the number of soap molecules $N_{\mathrm{S}}$ which in the present case was $N_{\mathrm{W}} / N_{\mathrm{S}}=66.6$. The composition of the continuous phase was determined by titration ${ }^{43}$ and it turned out that the ratio of benzene molecules $N_{\mathrm{O}}$ to the number of butanol molecules $N_{\mathrm{A}}$ is $N_{\mathrm{O}} / N_{\mathrm{A}}=4.2_{7} \pm 0.2$. The density of the continuous phase $\left(\rho=0.8572 \mathrm{~g} \mathrm{~cm}^{-3}\right)$, required in order to calculate the viscosity from a time-of-fall experiment, was measured with an Anton Paar DMA 10 digital densimeter. The viscosity of the continuous phase $(\eta=0.665 \mathrm{cP})$ was then determined with a HaakeKugelfall Precision model viscosimeter (falling ball type).

Systems with different concentrations of suspended particles were obtained by diluting an initially prepared microemulsion with its continuous phase. The volume fraction $\phi$ of the suspended particles was calculated from the mass of the material that constitutes the droplets assuming that there is no volume change on mixing. This volume fraction $\phi$ defines a radius $a$ through $\phi=4 \pi a^{3} n / 3$. The estimated errors on $\phi$ were $\approx 0.01$ for all concentrations. These errors are mainly due to the uncertainty in the distribution of the alcohol over the continuous and the suspended phase.

The quasi-elastic light scattering data were obtained with a Malvern 4300 photon correlation spectrometer. The light source was a $150 \mathrm{~mW}$ He-Ne laser (OIP model 181E). Data analysis was performed on-line with a HP 9825 A desk calculator. 


\section{RESULTS AND DISCUSSION}

In order to check whether the experimental data for $g_{2}(\tau)$ exhibit deviations from the single exponential form [eqn (15)] the data were fitted to the following expression using a non-linear least-squares procedure ${ }^{44}$

$$
g_{2}(\tau)=1+C \exp (-2 \Gamma \tau)\left(1+\mu_{2} \tau^{2}\right)
$$

Here $\mu_{2}$ is a measure of the deviation from the single exponential form. ${ }^{45} \mu_{2} / \Gamma^{2}$ lies in the range $0.02-0.04$, which is not significantly different from its estimated statistical uncertainty. This implies that the suspended particles are monodisperse and that the experimental data can be represented by eqn (15).

The diffusion coefficients calculated from $D=\Gamma / q^{2}$ are displayed in fig. 1 and listed in table 1. The initial decrease of $D$ with concentration can be represented empirically by

$$
D=D_{0}\left(1+k_{\mathrm{D}} \phi\right)
$$

with $D_{0}=4.4_{5} \pm 0.1 \times 10^{-7} \mathrm{~cm}^{2} \mathrm{~s}^{-1}$ and $k_{\mathrm{D}}=-5.6 \pm 0.2$.

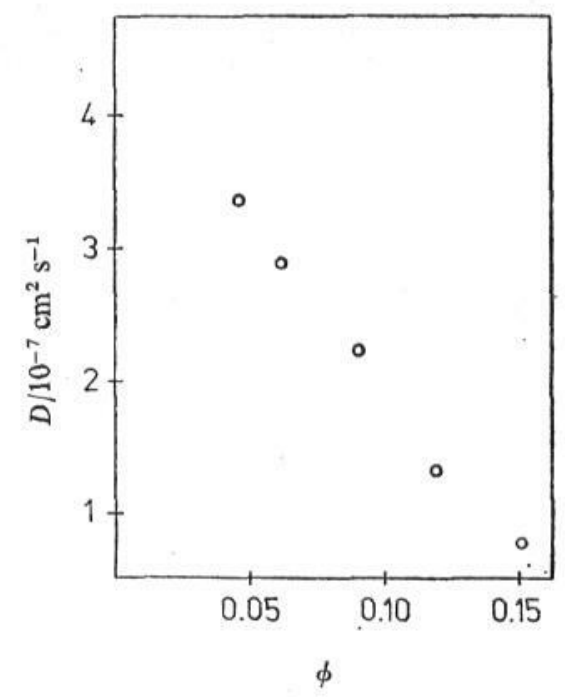

FIG. 1.-Diffusion coefficient as function of the volume fraction.

From the above value for $D_{0}$ and the viscosity of the continuous phase one obtains, using eqn (5), $a_{\mathbf{H}}=80 \pm 2 \AA$. If one assumes that the suspended particles are hard spheres with radius $a_{\mathrm{HS}}$, i.e.,

$$
W_{12}(r)= \begin{cases}\infty & \text { for } r<2 a_{\mathrm{HS}} \\ 0 & \text { for } r \geqslant 2 a_{\mathrm{HS}}\end{cases}
$$

one obtains $B_{2}^{\mathrm{HS}}=16 \pi a_{\mathrm{HS}}^{3} / 3$.

As mentioned in section 2 the theory for the concentration dependence of the mobility applies only if $a_{\mathrm{HS}} \geqslant a_{\mathrm{H}}$. Given this limitation the most reasonable assumption appears to be to put $a_{\mathrm{HS}}=a_{\mathrm{H}}$. One then obtains $\lambda^{\mathrm{HS}}=-6.55$. Finally assuming $a_{\mathrm{H}}=a$ the above results for the osmotic pressure and the mobility lead to ${ }^{12}$ $k_{\mathrm{D}}^{\mathrm{HS}}=1.45$. This result is clearly not in accordance with the experimental value. 
TABLE 1.-Diffusion COEFFiCIENT AS FUNCTION OF THE VOLUME FRACTION

\begin{tabular}{cc}
\hline volume fraction & $\begin{array}{c}\text { diffusion coefficient } \\
/ 10^{-7} \mathrm{~cm}^{2} \mathrm{~s}^{-1}\end{array}$ \\
\hline 0.046 & $3.3_{6} \pm 0.1$ \\
0.062 & $2.8_{8} \pm 0.1$ \\
0.091 & $2.23 \pm 0.06$ \\
0.119 & $1.31 \pm 0.02$ \\
0.151 & $0.77_{7} \pm 0.01$ \\
\hline
\end{tabular}

A possible explanation for the observed negative value for $k_{\mathrm{D}}$ is to assume the presence of attractive forces between the suspended particles. Indeed an analysis of eqn (6) and (7) indicates that an attractive potential lowers the osmotic compressibility more than it raises the mobility, the net result being a decrease of $k_{\mathrm{D}}$. A simple way to account for the effect of attractive interactions has been proposed by Batchelor. ${ }^{35}$ In this model one assumes that at a distance $2 a$ from the centre of a particle there are $\alpha \phi$ more particles than one expects on the basis of a uniform distribution. This amounts to assuming that the pair correlation function $g(r)$ to zero'th order in the density consists of a hard-sphere part $g^{\mathrm{HS}}(r)$ and an attractive part $g^{\mathrm{ATT}}(r)$

$$
g(r)=g^{\mathrm{HS}}(r)+g^{\mathrm{ATT}}(r)
$$

where

and

$$
g^{\mathrm{HS}}(r)= \begin{cases}0 & \text { for } 0 \leqslant r \leqslant 2 a \\ 1 & \text { for } r>2 a\end{cases}
$$

$$
g^{\mathrm{ATT}}(r)=\frac{\alpha a}{12} \delta(r-2 a)
$$

The coefficients $B_{2}$ and $\lambda$ now contain, in addition to the hard-sphere part given above, an attractive contribution

$$
\left.\begin{array}{l}
B_{2}^{\mathrm{ATT}}=-\frac{1}{2} \alpha \frac{4 \pi a^{3}}{3} \\
\lambda^{\mathrm{ATT}}=0.44 \alpha
\end{array}\right\} .
$$

This leads to $k_{\mathrm{D}}^{\mathrm{ATT}}=-0.56 \alpha$. Combining the hard-sphere and attractive contributions one then obtains

$$
D=D_{0}[1+(1.45-0.56 \alpha) \phi+\ldots] \text {. }
$$

Thus the attractive interactions in this model lead to a decrease of $D$ with concentration for values of $\alpha>2.6$. The observed value of $k_{\mathrm{D}}=-5.6$ corresponds to a value of $\alpha \simeq 13$. This implies for example for a volume fraction $\phi=0.05$, the number of excess particles is 0.65 .

The effect of attractive contributions to the potential is commonly accounted for by adding a van der Waals-type term to the second virial coefficient ${ }^{46}$

$$
B_{2}^{\mathrm{ATT}}=-\frac{a_{\mathrm{vdW}}}{k_{\mathrm{B}} T}
$$


where $a_{\mathrm{vdw}}$ is a constant related to the attractive part of the potential. Comparing eqn (17) and (18) we see that

$$
\frac{a_{\mathrm{vdW}}}{k_{\mathrm{B}} T}=\frac{1}{2} \alpha \frac{4 \pi a^{3}}{3} .
$$

Using this relation one obtains from the data for $a_{\mathrm{vdw}}$ given by Agterof et al. ${ }^{1}$ for a water-in-oil type microemulsion consisting of water, benzene, potassium oleate and hexanol, $\alpha=10.7$ both for $N_{\mathrm{w}} / N_{\mathrm{S}}=23.5$ and $N_{\mathrm{W}} / N_{\mathrm{S}}=49.5$. This value compares well with our result for $\alpha$.

In order to obtain a more specific measure of the attractive interaction that could lead to the observed value of $k_{\mathrm{D}}$, we consider a simple square-well potential,

$$
W_{12}(r)= \begin{cases}\infty & \text { for } 0 \leqslant r \leqslant 2 a \\ -y k_{\mathrm{B}} T & \text { for } 2 a<r \leqslant 2 a(1+x) \\ 0 & \text { for } \quad r>2 a(1+x) .\end{cases}
$$

For this model potential the attractive contribution to the second virial coefficient is of the form

$$
B_{2}^{\mathrm{ATT}}=-4\left[\mathrm{e}^{y}-1\right]\left[(x+1)^{3}-1\right] \frac{4 \pi a^{3}}{3} .
$$

In order to obtain an analytic expression for $\lambda^{\mathrm{ATT}}$ for this potential we have used in eqn (9) for $A(r)$ and $B(r)$ the accurate series expansions recently obtained by Felderhof 47

$$
A(r)+2 B(r)-3(1+a / r)=-\frac{15}{4}\left(\frac{a}{r}\right)^{4}+\frac{27}{8}\left(\frac{a}{r}\right)^{6}+\frac{75}{4}\left(\frac{a}{r}\right)^{7}+\mathcal{O}\left[\left(\frac{a}{r}\right)^{8}\right]
$$

yielding

$$
\lambda^{\text {ATT }}=\left(\mathrm{e}^{y}-1\right)\left(6 x^{2}+12 x+\frac{15}{8(1+x)}-\frac{9}{64(1+x)^{3}}-\frac{75}{256} \frac{1}{(1+x)^{4}}-\frac{369}{256}\right) .
$$

Combining these results one obtains for the coefficient $k_{\mathrm{D}}$

$$
k_{\mathrm{D}}=1.45+\left(\mathrm{e}^{y}-1\right)\left(-8 x^{3}-18 x^{2}-12 x+\frac{15}{8(1+x)}-\frac{9}{64(1+x)^{3}}-\frac{75}{256} \frac{1}{(1+x)^{4}}-\frac{369}{256}\right) .
$$

Clearly the two parameters $x$ and $y$ cannot be determined separately from the observed value of $k_{\mathrm{D}}$. The latter value can be recovered with, e.g.,

$$
x \simeq 0.25 \text { and } y \simeq 1
$$

or

$$
x \simeq 0.5 \text { and } y \simeq 0.5
$$

\section{CONCLUDING REMARKS}

In the present paper we give evidence that the observed decrease of $D$ with concentration can be explained by the thermo-hydrodynamic theory of Batchelor, summarized in section 2, using a potential of mean force consisting of a hard-sphere repulsion and an attractive term. The parameters for this potential are consistent with the results obtained from the osmotic compressibility as determined by static light scattering. 
In the analysis it has been assumed that the hydrodynamic radius of the suspended particles is equal to the hard-sphere radius, which in turn was taken to be equal to the radius that follows from the volume fraction. To what extent these assumptions affect the results of the analysis is hard to assess. In particular for the case that $a_{\mathrm{HS}}<a_{\mathrm{H}}$, i.e., penetrating particles, no theory is available for the mobility coefficient $\lambda$.

To draw more definite conclusions about the applicability of the Batchelor theory a systematic study of systems with different interaction potentials must be undertaken. Measurement of the diffusion coefficient by quasi-elastic light scattering is potentially a powerful technique to study such interaction forces.

This work has been supported by Interuniversitair Instituut voor Kernwetenschappen (Belgium).

${ }^{1}$ W. G. M. Agterof, J. A. J. Van Zomeren and A. Vrij, Chem. Phys. Letters, 1976, 43, 363.

${ }^{2}$ A. A. Calje, W. G. M. Agterof and A. Vrij, in Micellization, Solubilization and Microemulsions, ed. K. L. Mittal (Plenum Press, N.Y., 1977), vol. 2, p. 779.

${ }^{3}$ A. Vrij, E. A. Nieuwenhuis, H. M. Fijnaut and W. G. M. Agterhof, Faraday Disc. Chem. Soc., $1978,65,101$.

4 J. C. Brown, P. N. Pusey, J. W. Goodwin and R. H. Ottewil, J. Phys. A, 1975, 8, 664.

${ }^{5}$ G. D. J. Phillies, G. B. Benedek and N. A. Mazer, J. Chem. Phys., 1976, 65, 1883.

${ }^{6}$ A. A. Graciaa, J. Lachaise, P. Chabrat, L. Letamend, J. Rouch, C. Vancamps, M. Bourrel and C. Chambu, J. Phys. Letters, 1977, 38, 253.

${ }^{7}$ A. A. Graciaa and J. Lachaise, J. Phys. Letters, 1978, 39, 235.

${ }^{8}$ B. D. Fair, D. Y. Chao and A. M. Jamieson, J. Colloid Interface Sci., 1978, 66, 323.

${ }^{9}$ A. M. Cazabat, M. Lagues, D. Langevin, R. Ober and C. Taupin, Compt. rend. B, 1978, 287, 25.

${ }^{10}$ A. M. Cazabat, D. Langevin and A. Pouchelon, J. Colloid Interface Sci., to be published.

${ }^{11}$ H. M. Fijnaut, C. Pathmananoharan, E. A. Nieuwenhuis and A. Vrij, Chem. Phys. Letters, 1978, 59, 351.

12 G. K. Batchelor, J. Fluid Mech., 1976, 74, 1.

13 J. L. Aguirre and T. J. Murphy, J. Chem. Phys., 1973, 59, 1833.

${ }_{14}$ G. D. J. Phillies, J. Chem. Phys., 1974, 60, 976.

${ }^{15}$ G. D. J. Phillies, J. Chem. Phys., 1975, 62, 3925.

16 G. D. J. Phillies, J. Chem. Phys., 1977, 67, 4690.

17 J. L. Anderson and C. C. Reed, J. Chem. Phys., 1975, 64, 3240.

${ }^{18}$ A. R. Altenberger and J. M. Deutch, J. Chem. Phys., 1973, 59, 894.

19 A. R. Altenberger, Chem. Phys., 1976, 15, 269.

${ }^{20}$ W. Hess and R. Klein, Physica, 1976, 85A, 509.

${ }^{21}$ S. Harris, J. Phys. A, 1976, 9, 1895.

${ }^{22}$ B. J. Ackerson, J. Chem. Phys., 1976, 64, 242.

${ }^{23}$ B. J. Ackerson, J. Chem. Phys., 1978, 69, 684.

${ }^{24}$ B. U. Felderhof, J. Phys. A, 1978, 11, 929.

${ }^{25}$ S. A. Allison, E. L. Chang and J. M. Schurr, Chem. Phys., 1979, 38, 29.

${ }^{26}$ R. Zwanzig, Adv. Chem. Phys., 1969, 15, 325.

27 J. M. Deutch and I. Oppenheim, J. Chem. Phys., 1971, 54, 3547.

${ }^{28}$ T. J. Murphy and J. L. Aguirre, J. Chem. Phys., 1972, 57, 2098.

29 A. Einstein, Ann. Phys., 1905, 17, 549.

${ }^{30}$ A. Einstein, Z. Elektrochem., 1908, 14, 235.

${ }^{31}$ L. Landau and E. Lifshits, Physique Statistique (Mir, Moscow, 1967), p. 337.

${ }^{32}$ L. Landau and E. Lifshitz, Mécanique des Fluides (Mir, Moscow, 1971), p. 81.

${ }^{33}$ L. Landau and E. Lifshitz, Mécanique des Fluides (Mir, Moscow, 1971), p. 285.

${ }^{34}$ T. L. Hill, An Introduction to Statistical Thermodynamics (Addison Wesley, Reading, Mass., 1960), chap. 19.

${ }^{35}$ G. K. Batchelor, J. Fluid Mech., 1972, 52, 245.

${ }^{36}$ M. Stimson and G. B. Jeffery, Proc. Roy. Soc. A, 1926, 111, 110.

${ }^{37}$ A. J. Goldman, R. G. Cox and H. Brenner, Chem. Eng. Sci., 1966, 21, 1151.

${ }^{38}$ B. J. Berne and R. Pecora, Dynamic Light Scattering (Wiley, N.Y., 1976), chap. 13.

${ }^{39}$ P. N. Pusey, J. Phys. A, 1975, 8, 1433; 1978, 11, 119. 
${ }^{40}$ H. Z. Cummins and E. R. Pike, Photon Correlation and Light Beating Spectroscopy (Plenum Press, New York, 1974).

${ }^{41}$ L. Onsager, Phys. Rev., 1931, 37, 405.

${ }^{42}$ T. P. Hoar and J. H. Schulman, Nature, 1943, 102, 152.

43 J. E. Bowcot and J. H. Schulman, Z. Elektrochem., 1955, 59, 283.

${ }^{44}$ M. J. D. Powell, Comp. J., 1964, 7, 155.

${ }^{45}$ D. Koppel, J. Chem. Phys., 1972, 57, 4814.

${ }^{46}$ B. Widom, J. Chem. Phys., 1963, 39, 2808.

${ }^{47}$ B. U. Felderhof, Physica, 1977, 89A, 373.

(PAPER 9/1144) 